\title{
Arbeitswissenschaft und Globalisierung
}

\author{
Klaus J. Zink ${ }^{1}$ Manfred Schweres ${ }^{2}$ \\ Angenommen: 26. April 2021 / Online publiziert: 21. Mai 2021 \\ (c) Springer-Verlag GmbH Deutschland, ein Teil von Springer Nature 2021
}

Die Auseinandersetzung mit den „Systemgrenzen“ der Arbeitswissenschaft wurde schon in den 1970er und 1980erJahren eröffnet. So hat z. B. Schweres in der ZfA 1/1980 die Übertragbarkeit des Systemansatzes auf eine Theorie integrativ organisierter, interdisziplinär orientierter Arbeitswissenschaft erörtert, wobei sich die Diskussion zunächst auf das Mensch-Maschine bzw. Mensch-Technik Modell (Mikrosystem der Arbeit) konzentrierte. In Anlehnung an Fürstenberg $(1975,1978)$ hat er jedoch darauf hingewiesen, dass dies nicht übergreifende Probleme (z. B. Sozialorganisation) bzw. übergreifende Systeme umfasse, in denen Mensch-Maschine-Systeme eingebettet sind. In derart geschlossenen Systemen fehlt somit der Umweltbezug (Gesellschaftsbezug). In solchen Mikroarbeitssystemen wird in der Regel das System auch statisch betrachtet (Zeitpunktbezug). Aus dem Blick gerät dabei der dynamische Charakter menschlicher Arbeit (Prozesscharakter). Insofern bedarf es der Makrosysteme mit Umweltbezug (Schweres 1980). Diese Betrachtungsweise entspricht dem Konzept des sozio-technischen Systems (Zink 1997), wie es im Kontext des BMBF Förderprogramms „Humanisierung der Arbeit“ in den 1970er-Jahren mit Rückgriff auf die Studien des Tavistock Instituts wieder thematisiert wurde (Trist 1981). Mit der Erweiterung des Mensch-Maschine-Umwelt-Ansatzes entwickelte sich in den USA das Konzept Macroergonomics basierend auf der Analyse des Human-System In-

Wenn auch für ein Editorial eher unüblich, steht am Anfang ein kurzer Abriss wesentlicher Aspekte der Theorieentwicklung der neueren Arbeitswissenschaft, um den Lesenden die Positionsbestimmung zu verdeutlichen, von der aus die Mitwirkenden dieses Themenheftes ihre jeweiligen Beiträge erstellten. Daher auch ein Quellenverzeichnis am Ende.

Prof. Dr. Klaus J. Zink

kjzink@ita-kl.de

1 Institut für Technologie und Arbeit e. V., TU Kaiserslautern, Trippstadter Str. 113, 67663 Kaiserslautern, Deutschland

2 Institut für Fabrikanlagan und Logistik, Leibniz Universität Hannover, An der Universität 2, 30823 Garbsen, Deutschland terface (Hendrick und Kleiner 2002). Trotz dieser Weiterentwicklung war der Betrachtungsgegenstand immer noch ein Arbeitssystem in einer Organisation. Einer gewissen Erweiterung der Betrachtungsweise folgte das Memorandum der Gesellschaft für Arbeitswissenschaft „Die Zukunft der Arbeit erforschen“ (GfA 2000), in dem die Thematik der ,vernetzten Informations- und Arbeitswelt" aufgegriffen wurde. Dennoch stand auch hier weitgehend ein nationaler Fokus in der Theorieentwicklung im Vordergrund. In der jüngeren Vergangenheit verstärkten sich die Auswirkungen der EU-Einbindung Deutschlands zudem im Forschungsbereich, was allerdings in der Regel ,nur“ bedeutete, dass gleichartige Problemstellungen in unterschiedlichen Nationen gleichzeitig bearbeitet wurden. Die Notwendigkeit der Betrachtung der Arbeitsbedingungen in internationalen Beschaffungsketten (Zink 2014a, 2017) wurde eher durch Konzepte wie „Corporate Social Responsibility“ und die daraus weltweit resultierenden Gesetzgebungen initiiert, weil sich zeigte, dass die zunächst angestrebte Freiwilligkeit der Übernahme gesellschaftlicher Verantwortung durch die Unternehmen nicht zum Erfolg führte. Aus arbeitswissenschaftlicher Sicht macht dies eine System-of-SystemsBetrachtungsweise erforderlich (Thatcher und Yeow 2016; Zink 2014b), d.h. man muss Arbeitssysteme in unterschiedlichen Unternehmen und Kontexten entlang der Lieferkette betrachten. Wenn man diese zusätzliche Perspektive benennen will, kann man z.B. von "Supply-Chain-Ergonomics“" reden (Zink 2017). Dabei muss man konstatieren, dass Globalisierung kein neues Phänomen ist, aber in der (internationalen) Arbeitswissenschaft bisher keine wesentliche Rolle gespielt hat. Die Sensibilisierung für nicht akzeptable Arbeitsbedingungen hat (national und international) jedoch zugenommen und den Ruf nach einer gesellschaftlichen Verantwortung von Unternehmen für Ihr Handeln in der Lieferkette verstärkt. Dies schlägt sich in einer Reihe von Resolutionen und Gesetzen bzw. Gesetzentwürfen nieder, die es bis dahin nicht gab. Beispielhaft seien die UN Initiative „Business and Human Rights“ (OHCR 2011) und deren Umsetzung in nationale Aktionspläne, die Vorhaben der Europäischen Union (European Commission 2020) so- 
wie die deutsche Initiative zu einem „Lieferkettengesetz“ (BMAS 2021) genannt. In diesem Kontext stellt sich die Frage, ob diese Entwicklung auch Chancen für eine Weiterentwicklung der Arbeitswissenschaft bietet.

In diesem Themenheft sollen daher einige Aspekte aufgegriffen werden, die eine Basis für eine weiterführende Diskussion bieten können. ${ }^{1}$ Die Relevanz dieses Themas wird in einem ersten Beitrag von Hiba, Jentsch \& Zink insbesondere am Beispiel von Sonderwirtschaftszonen dargestellt. Hiba zeigt die Aktivitäten der International Labour Organization (ILO) auf diesem Gebiet, während Jentsch am Beispiel der Textilindustrie veranschaulicht, dass diese und andere Maßnahmen nur begrenzt erfolgreich waren. Zink diskutiert kritisch potenzielle Weiterentwicklungen in den Sonderwirtschaftszonen. Um die menschengerechte Gestaltung guter Arbeit in internationalen Beschaffungsketten voranzubringen und neuen Arbeitsund Beschäftigungsformen gerecht zu werden, bedarf es aber künftig entsprechender (arbeits- und sozial-)rechtlicher Rahmenbedingungen, die auch für die Arbeitswissenschaft relevant sind. Damit setzen sich in diesem Themenheft Heinen, Kohte und Heilmann auseinander: Heinen beleuchtet die arbeitsrechtlichen Aspekte in internationalen Lieferketten, Kohte diskutiert den Arbeits- und Gesundheitsschutz im Rahmen der Arbeitnehmerentsendung, während Heilmann sich grundsätzlich mit dem Änderungsbedarf im (nationalen) Arbeitsrecht beschäftigt, der sich durch Digitalisierung, Entgrenzung und generell eine Arbeit im Wandel ergibt. Eine europäische Perspektive auf den Arbeits- und Gesundheitsschutz, die Impulse für eine globaler orientierte Arbeitswissenschaft liefern kann, stellen Windel und HausRybicki dar. Während Globalisierung in der Vergangenheit eher mit der Auslagerung einfacher, arbeitsintensiver Tätigkeiten (wie z.B. in der Textilindustrie) verbunden war, betrifft das in den letzten Jahren auch vermehrt die Wissensarbeit. Dabei geht es nicht mehr nur um Kooperation mit Sonderwirtschaftszonen oder einzelnen Organisationen wie Foxconn in Taiwan, sondern um die Aktivierung einzelner Personen weltweit. Dieser auch als Crowdsourcing bezeichnete Ansatz wird am Beispiel der Aktivitäten der IG Metall beleuchtet. Bertolini, Borkert, Ferrari \& Graham setzen sich mit der Frage auseinander. wie gute oder faire Arbeit im Rahmen der sogen. Gig-Economy bzw. Plattformökonomie bewertet werden kann und zeigen das anhand der „Fair-Work-Initiative“ des Oxford Internet Institute. Bengler, Schmauder und Zink versuchen in einem

\footnotetext{
1 Z. B. im Zusammenhang mit einem Forschungsprojekt „Arbeitswissenschaft und globale Lieferketten - ein potenzieller Beitrag zu einer sozialen Marktwirtschaft in Zeiten der Globalisierung “ am Institut für Technologie und Arbeit e. V. an der TU Kaiserslautern, in dessen Kontext dieses Themenheft entstanden ist. Dieses Projekt wird von der Wolfgang Schultze Stiftung für soziale Verantwortung in der Marktwirtschaft finanziell unterstützt.
}

abschließenden Beitrag, potenzielle Konsequenzen für die Arbeitswissenschaft zu diskutieren: Schmauder weist auf die Instrumente hin, die die Arbeitswissenschaft jetzt schon Unternehmen zur Verfügung stellen kann, um ihrer Verantwortung in der Lieferkette im Hinblick auf menschengerechte Arbeitsbedingungen besser gerecht zu werden. Zink diskutiert die Notwendigkeit der Integration neuer Zielgruppen am Beispiel Beschaffung und der daraus resultierenden Konsequenzen für die arbeitswissenschaftliche Ausund Weiterbildung (Schweres und Sengotta 1997). Bengler stellt abschließend fest, dass eine globalere Perspektive der Arbeitswissenschaft zunächst mit einer Intensivierung europäischer Forschungsprogramme beginnen sollte. Ein abschließendes Zwischen-Fazit soll die Grundlage für weiterführende Diskussionen bilden.

Kaiserslautern und Duisburg im März 2021

Klaus J. Zink und Manfred Schweres

\section{Literatur}

BMAS (Bundesministerium für Arbeit und Soziales) (Hrsg.) (2021) Sorgfaltspflichtengesetz. https://www.bmas.de/DE/Service/presse/ Pressemitteilung/2021/bundeskabinett-verabschiedetsorgfaltspflichtengesetz.html. Zugegriffen: 28 Mär 2021

European Commission (Ed.) (2021) Study on due dilligence requirements through the supply chain, Brussels. https://op.europa.eu/ en/publication-detail/-publication/291b84d3-4c82-11ea-b8b701aa75ed71a1/language-en/format-PDF/source-search. Zugegriffen: 7 Jan 2021

Fürstenberg F (1975) Konzeption einer interdisziplinär organisierten Arbeitswissenschaft. Kommission für wirtschaftlichen und sozialen Wandel, Bd. 64. Schwartz, Göttingen

Fürstenberg F (1978) Integrative Arbeitswissenschaft - Eine Alternative zum Taylorismus. In: Pornschlegel H, Scholz H (Hrsg) Arbeitswissenschaft in der Gesellschaftspolitik. Duncker \& Humblot, Berlin

GfA (Gesellschaft für Arbeitswissenschaft e. V.) (Hrsg) (2000) Die Zukunft der Arbeit erforschen. Ein Memorandum der Gesellschaft für Arbeitswissenschaft e. V. zum Strukturwandel der Arbeit. GfA Press, Dortmund

Hendrick HW, Kleiner BM (2002) Macroergonomics: theory, methods, and applications. Lawrence Erlbaum, Mahwah

OHCR (UN Commision on Human Rights) (Ed.) (2021) Guising Principles on Business and Human Rights. https://www.ohcr.org/ en/issues/business/pages/businessindex.aspx. Zugegriffen: 7 Jan 2021

Schweres M (1980) Strukturelemente einer integrativen Arbeitswissenschaft. Z Arb Wiss 34(1):1-12

Schweres M, Sengotta M (1997) Arbeitswissenschaftliche Qualifizierung: Angebots- und Nachfragestrukturen. In: Luczak W, Volpert W (Hrsg) Handbuch der Arbeitswissenschaft. Schäffer-Poeschel, Stuttgart, S 40-46

Thatcher A, Yeow PHP (2016) A sustainable system of systems approach: a new HFE paradigm. Ergonomics 59(2):167-178

Trist EL (1981) The evolution of sociotechnical systems. Issues in the quality of working life. Occasional Papers No. 2. Ontario Quality of Working Life Centre, Toronto

Zink KJ (1997) Soziotechnische Ansätze. In: Luczak W, Volpert W (Hrsg) Handbuch der Arbeitswissenschaft. Schäffer-Poeschel, Stuttgart, S 74-77 
Zink KJ (2014a) Arbeitswissenschaft und globales Umfeld. Z Arb Wiss 68(4):203-207

Zink KJ (2014b) Designing sustainable work systems: the need for a systems approach. Appl Ergon 45(1):126-132

Zink KJ (2017) Nachhaltige Arbeitssysteme in (internationalen) Wertschöpfungsketten. Z Arb Wiss 71(2):92-100 